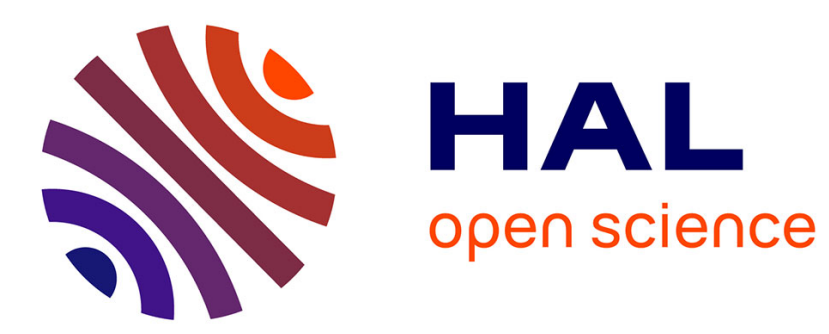

\title{
Principes pour un procédé industriel de fractionnement des protéines du lactosérum
}

\author{
A. Pierre, J. Fauquant
}

\section{To cite this version:}

A. Pierre, J. Fauquant. Principes pour un procédé industriel de fractionnement des protéines du lactosérum. Le Lait, 1986, 66 (4), pp.405-419. hal-00929078

\section{HAL Id: hal-00929078 \\ https://hal.science/hal-00929078}

Submitted on 1 Jan 1986

HAL is a multi-disciplinary open access archive for the deposit and dissemination of scientific research documents, whether they are published or not. The documents may come from teaching and research institutions in France or abroad, or from public or private research centers.
L'archive ouverte pluridisciplinaire HAL, est destinée au dépôt et à la diffusion de documents scientifiques de niveau recherche, publiés ou non, émanant des établissements d'enseignement et de recherche français ou étrangers, des laboratoires publics ou privés. 


\title{
Principes pour un procédé industriel de fractionnement des protéines du lactosérum
}

\author{
A. PIERRE et J. FAUQUANT \\ I.N.R.A., Laboratoire de Recherches de Technologie laitière \\ 65 , rue de Saint-Brieuc, 35042 Rennes cedex, France
}

\section{Résumé}

Les propriétés d'agrégation de l'a-lactalbumine, sous l'effet d'un traitement thermique modéré, à $\mathrm{pH}$ acide ont été utilisées pour mettre au point un procédé, extrapolable industriellement, de fractionnement des composants protéiques majeurs du lactosérum. Ce procédé met en œuvre :

- une clarification basée sur la déstabilisation thermocalcique des phospholipoprotéines ;

- la concentration par ultrafiltration du lactosérum clarifié ;

- la polymérisation à bas $\mathrm{pH}$ de l'o-lactalbumine et sa séparation après gélification thermique.

L'optimisation de chacune de ces étapes était étudiée tant en ce qui concerne la purification des protéines que l'obtention d'un rendement maximal.

La $\beta$-lactoglobuline était obtenue à $98 \%$ de pureté avec un rendement de l'ordre de $90 \%$. Par contre, la technologie développée ne permettait pas de séparer l'alactalbumine de la bovine sérum albumine. La fraction contenant ces deux protéines avait la composition suivante : $\alpha$-lactalbumine $50 \mathrm{~g} \%$ - Bovine sérum albumine $15 \mathrm{~g} \%$ - protéines non identifiées $13 \mathrm{~g} \%$.

Mots clés : Lactosérum - Protéines - Fractionnement - $\beta$-lactoglobuline - $\alpha$-lactalbumine - Purification.

\section{Summary}

Industrial process for production of purified proteins from whey

Aggregative properties of $\alpha$-lactalbumin under moderate heat treatment at low $\mathrm{pH}$ were used to develop a process, with potential industrial scale-up, for separating main protein components of whey. This process includes the following steps :

- clarification of the whey by a combination of a calcium ions addition and of heat treatment which destabilize the phospholipoproteins;

- UF concentration of the clarified whey ;

- polymerisation at low $\mathrm{pH}$ of $\alpha$-lactalbumin and its separation after thermal gelification.

Optimization of each step was studied in order to improve purity of separated protein and to get maximal yield. 
The obtained $\beta$-lactoglobulin fraction was $98 \%$ pure with a yield of approximately $90 \%$. But, in the proposed process $\alpha$-lactalbumin and bovine serum albumin were not separated. The corresponding fraction had the following composition (w/w) $\alpha$-lactalbumine $50 \%$ - Bovine serum albumine $15 \%$ - Unidentified proteins $13 \%$.

Key words : Whey - Proteins - Fractionation - B-lactoglobulin - $\alpha$-lactalbumin Purification.

\section{Introduction}

Les protéines du lactosérum ont une très haute valeur nutritionnelle et d'excellentes propriétés fonctionnelles. Elles constituent, à l'état concentré, des sources de protéines recherchées tant pour l'alimentation des jeunes animaux que pour l'alimentation humaine (MAUBOIS, 1982). Leur fragmentation en réacteur enzymatique à membrane (ROGER, 1979) conduit à des produits peptidiques correspondant parfaitement aux besoins de la diététique de réanimation entérale (MAUBOIS, 1984).

Du fait de leur disponibilité, de leur coût modéré (en regard de celui des autres sources de protéines animales) et de leurs caractéristiques, les protéines de lactosérum dans leur ensemble peuvent prétendre à une large utilisation dans les industries alimentaires. Cependant, l'accumulation des connaissances sur les propriétés de chacune des protéines qui les composent amène de plus en plus à envisager leur séparation en vue d'utilisations et de valorisations différenciées. Cette recherche de fractionnement concerne aussi bien les constituants protéiques majeurs ( $\beta$-lactoglobuline, $\alpha$-lactalbumine, immunoglobulines) que les constituants dits mineurs tels que la lactopéroxydase, la lactoferrine, en raison de leurs propriétés bactériostatiques, ou certains fragments de nature peptidique ayant une activité morphinomimétique (BRANTL et TESCHEMACHER, 1982).

De nombreuses méthodes ont été proposées pour séparer au niveau du laboratoire les constituants protéiques du lactosérum. La plupart ne sont pas extrapolables au plan industriel parce que longues, complexes, délicates à mettre en cuvre sur de grands volumes, coûteuses en énergie, de rendement faible et ignorant délibérément toute valorisation possible des co-produits, soit du fait de 1'utilisation de traitements thermiques intenses (NIELSEN et al., 1973) ou de pH très alcalins (HARris et Yoell, 1985), soit du fait de l'utilisation de sels (Kumata, 1985).

L'utilisation de résines échangeuses d'ions conduit, certes, à des produits de qualité et de pureté satisfaisantes (MIRABEL, 1978 ; SKUDder, 1985), mais elle nécessite des investissements élevés et amène, dans la plupart des cas, à une dilution non négligeable des protéines éluées, d'où un accroissement des coûts de concentration et de séchage.

La séparation thermique à bas pH telle qu'elle est décrite par PEARCE (1983) nous apparaît, au contraire, comme beaucoup plus séduisante pour la séparation de la $\beta$-lactoglobuline et de l' $\alpha$-lactalbumine, la première protéine étant obtenue à l'état soluble et la seconde sous forme d'un précipité. Mais, l' $\alpha$-lactalbumine ayant la propriété de s'associer aux constituants lipoprotéiques du lactosérum - selon Brown (1984) une mole d' $\alpha$-lactalbumine peut fixer, à pH 4,0, jusqu'à 
80 moles de phosphatidylcholine - il est probable que le précipité d' $\alpha$-lactalbumine obtenu selon le procédé décrit par PEARCE contenait les lipides résiduels du lactosérum. Or, ces substances sont le plus souvent gênantes aussi bien pour les opérations ultérieures de concentration purification (MAUBOIs, 1984) que pour celles de fragmentation (ROGER, 1979) en réacteur enzymatique à membrane.

La séparation des lipoprotéines, préalable à toute opération de fractionnement des protéines du lactosérum, étant facilement réalisable par les procédés décrits par Fauquant et al. (1985) et Fauquant et Pierre (1985), nous avons entrepris cette étude dans le but de mettre au point un procédé optimisé de préparation, sous forme purifiée, des deux protéines principales : la $\beta$-lactoglobuline et l' $\alpha$-lactalbumine. Chaque paramètre du protocole mis au point, basé sur les observations de PEARCE (1983) et sur nos propres observations, a été systématiquement étudié.

\section{Matériel et méthodes}

- Lactosérum utilisé. Il provenait d'une fromagerie d'Emmental et était débourdé et écrémé à $50^{\circ} \mathrm{C}$ avant expérimentation. Les quantités mises en œuvre variaient de quelques litres à plus de $1000 \mathrm{l}$. La clarification était réalisée par agrégation thermocalcique des phospholipoprotéines comme décrit par FAUQUANT et al. (1985) : le lactosérum froid $\left(2^{\circ} \mathrm{C}\right)$ était additionné de calcium (sous forme de $\mathrm{CaCl}_{2}$ ) jusqu'à une concentration totale de $1,2 \mathrm{~g} / \mathrm{l}$; le $\mathrm{pH}$ était ajusté à 7,3 et le lactosérum traité thermiquement $\left(79^{\circ} \mathrm{C}-20 \mathrm{~s}\right)$. Le précipité formé était séparé par simple décantation puis filtration du surnageant sur filtre Seitz équipé de membranes filtrantes Super 80. La clarification était appréciée par mesure de la densité optique à $600 \mathrm{~nm}$. Lorsqu'une modification du $\mathrm{pH}$ du lactosérum était nécessaire, le $\mathrm{pH}$ était ajusté avec de l'acide chlorhydrique concentré ou de la soude $10 \mathrm{~N}$.

- Ultrafiltration. Une unité pilote comportant une pompe volumétrique à débit variable et une cartouche ROMICON ayant une surface membranaire de $1,5 \mathrm{~m}^{2}$ (membrane PM 50 ou PM 10) était utilisée. Les volumes de lactosérum soumis à ultrafiltration étaient de l'ordre de 200 litres.

- Microfiltration. La même unité pilote était utilisée mais avec remplacement de la cartouche ROMICON par un module SFEC équipé de membranes M6 1000 ayant une surface de $0,113 \mathrm{~m}^{2}$.

- Traitements thermiques. Au laboratoire, le traitement thermique des rétentats d'ultrafiltration était réalisé sur $10 \mathrm{ml}$ de la solution à tester contenue dans un tube à essais de $18 \times 180$ et portée à $56^{\circ} \mathrm{C}$ pendant 30 min (en bain d'eau thermostaté). Lorsqu'il y avait formation d'un gel translucide, ce dernier était dispersé dans $10 \mathrm{ml}$ d'eau ajoutée dans le tube à essais.

$\mathrm{Au}$ niveau des expérimentations pilotes, le traitement thermique du concentré de lactosérum était réalisé dans un bain d'eau thermostaté. 
Déterminations des teneurs en protéines. Les teneurs en $\beta$-lactoglobuline, en $\alpha$-lactalbumine et en sérum-albumine étaient déterminées par HPLC, en gel filtration, comme décrit par FAUQUANT et al. (1985), sur les surnageants obtenus par centrifugation (1000 g, $10 \mathrm{~min}$ ) des suspensions de gels dispersés dans l'eau et dilués en tampon Tris- $\mathrm{HCl} 0,01 \mathrm{M}, \mathrm{pH}$ 6,68. Les quantités de protéines précipitées par le traitement thermique étaient appréciées par différence entre les valeurs déterminées sur le produit initial et sur le surnageant.

Electrophorèses. Les électrophorèses étaient réalisées comme décrit par ANDREWS (1983).

Lorsque des rétentats de lactosérum obtenus par ultrafiltration seront utilisés, leur concentration sera évaluée par la teneur en $\alpha$-lactalbumine, étant sous-entendu qu'ils contiennent toutes les autres protéines du lactosérum, à des teneurs augmentées dans la même proportion.

\section{Résultats et discussion}

Pour le fractionnement du lactosérum, nous avons mis en œuvre les étapes technologiques suivantes : une clarification, une concentration par ultrafiltration, une polymérisation de l' $\alpha$-lactalbumine contenue dans le rétentat UF à bas $\mathrm{pH}$, suivie d'un traitement thermique entraînant la gélification, enfin, la séparation des fractions protéiques obtenues et leur purification. Chaque traitement a fait l'objet d'une étude de paramètres destinée à optimiser le procédé.

\section{A. Obtention d'un rétentat de lactosérum clarifié}

\section{Clarification du lactosérum}

L'application au lactosérum brut du traitement proposé par FAUQUANT et al. (1985) entraînait une forte réduction du pic d'exclusion, lors de l'analyse par HPLC, comme le montre la comparaison des profils chromatographiques (fig. 1) avant et après traitement suivi d'une séparation par centrifugation. Ce pic d'exclusion (V.E. : 4,5) correspond pour l'essentiel aux phospholipoprotéines. Leur élimination entraîne un abaissement notable de la densité optique du lactosérum à $600 \mathrm{~nm}$, qui passait de la valeur 0,50 à la valeur 0,02 ce qui correspond alors à la limpidité de l'ultrafiltrat de lait ou de lactosérum.

La figure 1 montre également que le traitement de clarification ne modifiait pas les teneurs en $\beta$-lactoglobuline (V.E. : 6,9), en $\alpha$-lactalbumine (V.E. : 7,9) et en sérum albumine bovine (V.E. : 5,6). Par contre, ce traitement entraînait l'élimination des immunoglobulines (fig. 2) qui co-précipitaient avec les lipoprotéines.

\section{Ultrafiltration du lactosérum clarifié}

Le lactosérum clarifié était acidifié à $\mathrm{pH} 3,8$, par addition d'acide chlorhydrique, dans le but de favoriser un début de polymérisation de l' $\alpha$-lactalbumine 


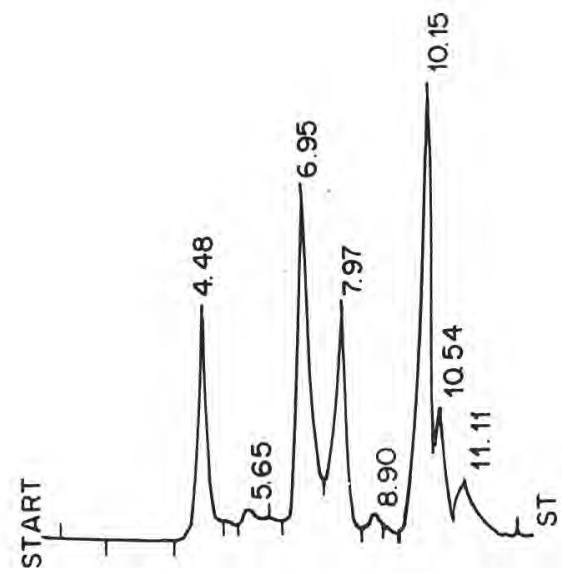

Lactoserum initial

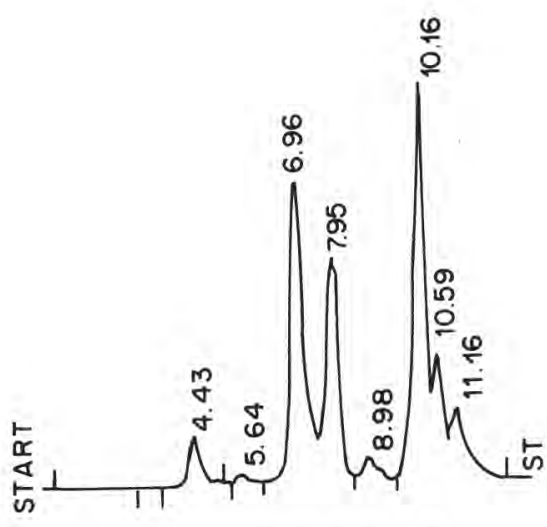

Loctosérum clarifié

Fig. 1

Profil d'élution en HPLC du lactosérum initial et clarifié, VE : 4,4 phospholipoprotéines ; 6,9 -lactoglobuline ; 7,9 $\alpha$-lactalbumine; 10,0 sels et vitamines.

Fractionation by HPLC of proteins in whey before and after clarifying treatment. EV : 4.4

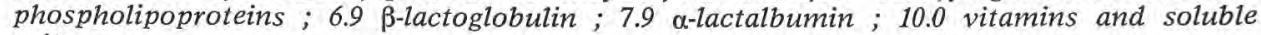
salts.

puis une concentration en volume de 30 fois était réalisée. Deux types de membranes ROMICON PM 50 et PM 10 (seuil de coupure 50000 et 10000 ) ont été comparés. La membrane PM 50 permet d'obtenir des débits doubles de la PM 10, mais, du fait de la taille des pores de ce type de membranes, un passage d' $\alpha$-lactalbumine était constaté dans le perméat; il représentait $20 \%$ de l' $\alpha$-lactalbumine présente dans le lactosérum clarifié mis en œuvre.

La clarification du lactosérum accroissait notablement les débits de perméation. Toutes choses étant égales par ailleurs, le débit de perméation initial observé avec le lactosérum clarifié était égal à 1,8 fois celui observé avec le même lactosérum brut dans l'installation expérimentale utilisée, soit $90 \mathrm{l} / \mathrm{h} / \mathrm{m}^{2}$ au lieu de $50 \mathrm{l} / \mathrm{h} / \mathrm{m}^{2}$. Cet accroissement des débits de perméation comme l'accroissement de la perméation des protéines de la taille de l' $\alpha$-lactalbumine est à mettre au compte de l'élimination des lipoprotéines, principaux composants responsables de la formation de la couche de polarisation des membranes d'ultrafiltration et donc de leur colmatage (MAUBoIs, 1982).

\section{B. Précipitation sélective des protéines par traitement thermique}

Cinq paramètres (température et durée du chauffage, $\mathrm{pH}$, teneur en protéines du rétentat, nature de l'acide utilisé) ont été étudiés pour accroître la poly- 


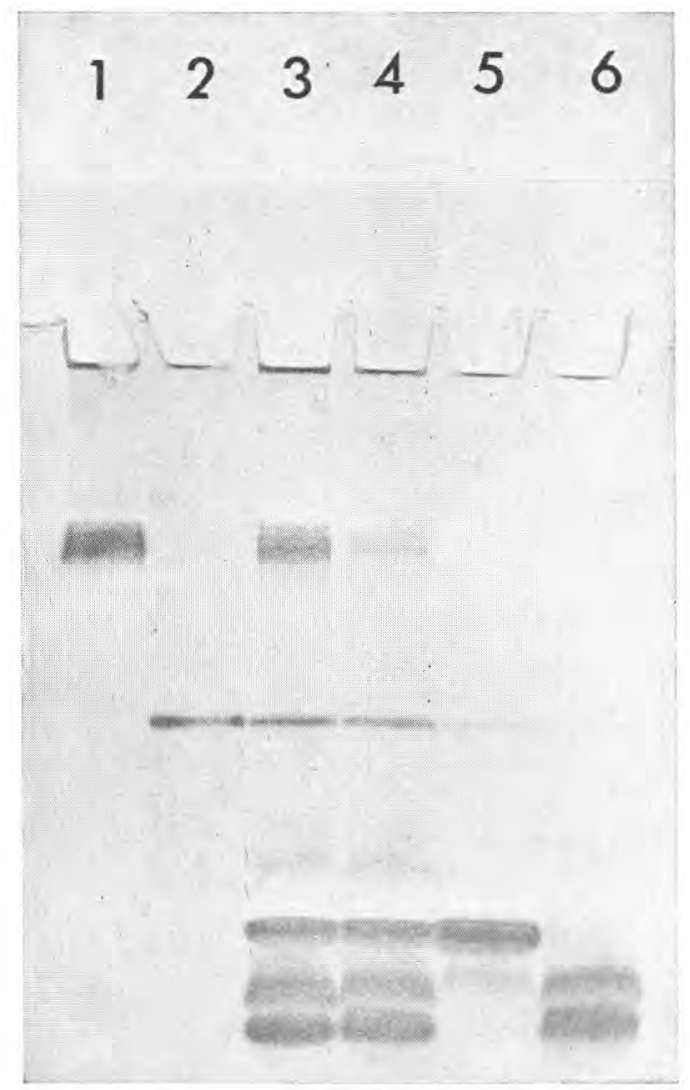

Fig. 2

Séparation par électrophorèse des constituants du lactosérum avant (3) et après (4) clarification. Protéines de référence : 1. Immunoglobulines; 2 . Bovine sérum albumine; 5. $\alpha$-lactalbumine; 6. $\beta$-lactoglobuline.

Fractionation of whey proteins by electrophoresis before (3) and after (4) clarifying treatment. Reference proteins : 1. Immunoglobulins ; 2. Bovine serum albumine ; 5 . $\alpha$-lactalbumine ; 6. $\beta$-lactoglobulin.

mérisation de $1^{\prime} \alpha$-lactalbumine à bas $\mathrm{pH}$ et sa gélification (KRONMAN et al., 1964, PEARCE, 1983).

\section{Effet de la température}

Le test de précipitation (cf. Matériel et méthodes) était réalisé à $45^{\circ} \mathrm{C}$, $55^{\circ} \mathrm{C}$ et $65^{\circ} \mathrm{C}$ pendant $30 \mathrm{~min}$ sur un rétentat ayant une teneur en $\alpha$-lactalbumine égale à $21 \mathrm{~g}$ par $\mathrm{kg}$. A $45^{\circ} \mathrm{C}$, aucune précipitation n'était observée. Par contre, à $55^{\circ} \mathrm{C}$ et $65^{\circ} \mathrm{C}$, les rendements de précipitation étaient respectivement de $78 \%$ et de $88 \%$. Ces résultats ne sont pas directement comparables à ceux de PeARCE (1983) qui, lui, avait mesuré la proportion d'azote total précipité. 


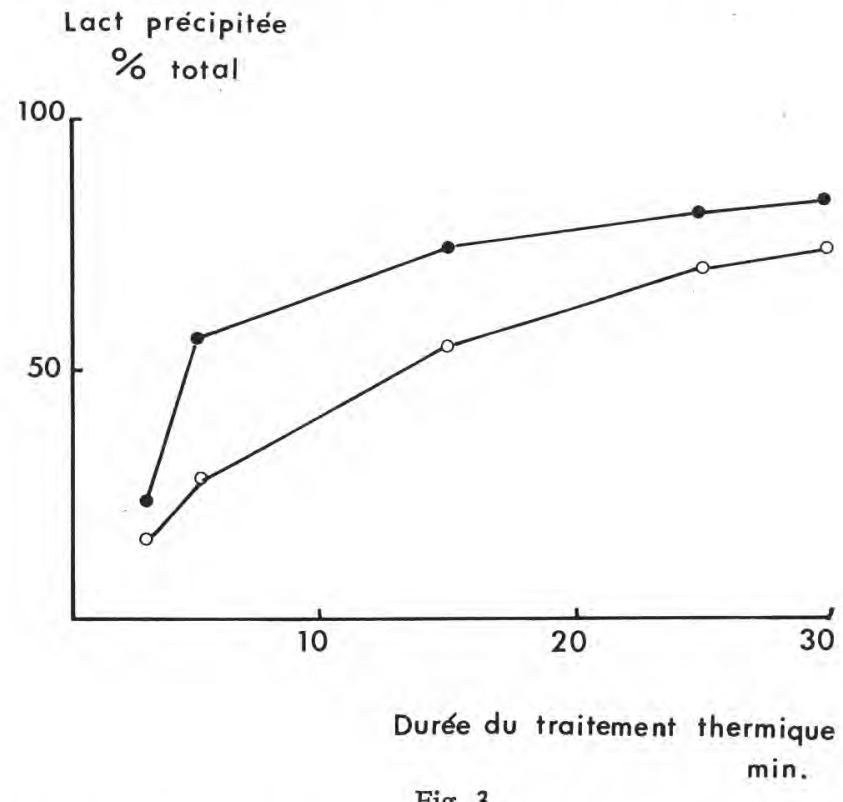

Fig. 3

Influence de la concentration du lactosérum sur la proportion d'a-lactalbumine précipitée par le traitement thermique.

Incidence of protein concentration in whey on precipitation yield of a-lactalbumin on heating.

\section{Influence de la durée du traitement thermique}

La figure 3 montre la progression de la précipitation au cours du temps pour deux rétentats contenant respectivement $10 \mathrm{~g}$ et $20 \mathrm{~g}$ d' $\alpha$-lactalbumine par $\mathrm{kg}$. L'accroissement du rendement de précipitation était surtout important au cours des 20 premières minutes; entre 25 et $30 \mathrm{~min}$, l'influence du temps devenait faible. La polymérisation de l' $\alpha$-lactalbumine et la gélification des lactosérums concentrés sont donc des phénomènes relativement lents. La proportion d' $\alpha$-lactalbumine précipitée en $30 \mathrm{~min}$ était de $75 \%$ dans le rétentat à $10 \mathrm{~g} / \mathrm{kg}$ et de $85 \%$ dans le rétentat à $20 \mathrm{~g} / \mathrm{kg}$.

\section{Effet du $p H$}

Les rendements de précipitation de l' $\alpha$-lactalbumine ont été comparés à différents $\mathrm{pH}($ de 3,3 à 5,5) à partir de rétentats contenant de 6 à $20 \mathrm{~g}$ d' $\alpha$-lactalbumine par $\mathrm{kg}$. L'ultrafiltration ayant été réalisée à $\mathrm{pH} 3,8$, les $\mathrm{pH}$ étaient ajustés à la valeur désirée soit par addition d'acide chlorhydrique, soit par addition de soude. Les résultats rassemblés dans la figure 4 montrent qu'il existe un optimum de précipitation aux environ de $\mathrm{pH} 3,8$ pour les rétentats UF ayant des teneurs en $\alpha$-lactalbumine supérieures à $12 \mathrm{~g} / \mathrm{kg}$. Les rétentats moins concentrés précipitent seulement aux $\mathrm{pH}$ supérieurs à 4,2 . 


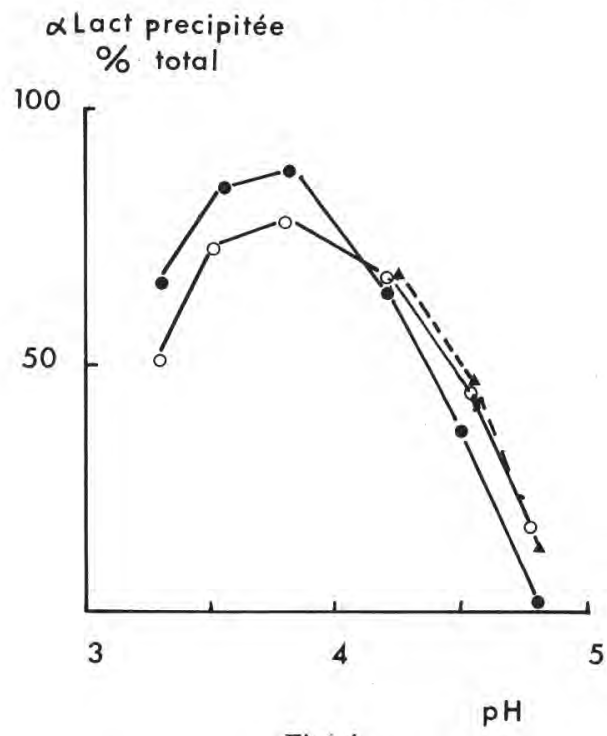

Fig. 4

Influence $d u$ pH du lactosérum et de sa concentration sur la proportion d'a-lactalbumine précipitée par le traitement thermique. $\alpha-L 20(\bullet), 12(0), 9 \mathrm{~g} / \mathrm{kg}(\mathbf{\Delta})$.

Incidence of $\mathrm{pH}$ and protein concentration of whey on precipitation yield of $\alpha$-lactalbumin on heating. $\alpha-L 20(\bullet), 12(0), 9(\Delta) \mathrm{g} / \mathrm{kg}$.

Ces résultats sont à comparer avec ceux de PeARCE (1983) qui observait une précipitation de l' $\alpha$-lactalbumine même dans le lactosérum non ultrafiltré et non clarifié. Il semble donc que la clarification thermo-calcique élimine un adjuvant de précipitation de l' $\alpha$-lactalbumine constituant une impureté dans le précipité.

L'aspect du précipité formé était très dépendant du $\mathrm{pH}$. Aux $\mathrm{pH}$ inférieurs à 4,0 , le gel formé était translucide et très ferme. Son volume diminuait peu lors de la centrifugation, ce qui indique une hydrophilie élevée. Par contre, aux $\mathrm{pH}$ supérieurs à 4,0, il se formait un précipité blanc, opaque, souple et fortement rétractable lors de la centrifugation.

\section{Effet de la nature de l'acide}

L'acidification du lactosérum clarifié par l'acide nitrique a été comparée à celle obtenue par addition d'acide chlorhydrique. Le rétentat contenant $16 \mathrm{~g}$ d' $\alpha$-lactalbumine par $\mathrm{kg}$ était amené à $\mathrm{pH} 3,5,3,8$ et 4,2 et chauffé à $55^{\circ} \mathrm{C}$ pendant $30 \mathrm{~min}$. Les rendements de précipitation de l' $\alpha$-lactalbumine étaient en moyenne supérieurs de $10 \%$ à ceux observés avec un rétentat identique amené aux mêmes $\mathrm{pH}$ par l'acide chlorhydrique $(87 \%$ contre $77 \%$ à $\mathrm{pH} 3,5 ; 82 \%$ contre $75 \%$ à pH 3,8;67\% contre $55 \%$ à $\mathrm{pH} 4,2$ ).

L'ion nitrate favorise la polymérisation. KRONMAN (1964) avait déjà montré que la nature des anions mis en présence de l' $\alpha$-lactalbumine avait une influence sur sa stabilité. 


\section{Influence de la teneur en protéines du rétentat}

Plus le rétentat de lactosérum était concentré, plus élevé était le rendement de précipitation de l' $\alpha$-lactalbumine. La figure 5 montre que ce rendement augmente de $67 \%$ à $85 \%$ lorsque les teneurs en $\alpha$-lactalbumine et en $\beta$-lactoglobuline des rétentats passent respectivement de 6 et $38 \mathrm{~g} / \mathrm{kg}$ à 21 et $132 \mathrm{~g} / \mathrm{kg}$.

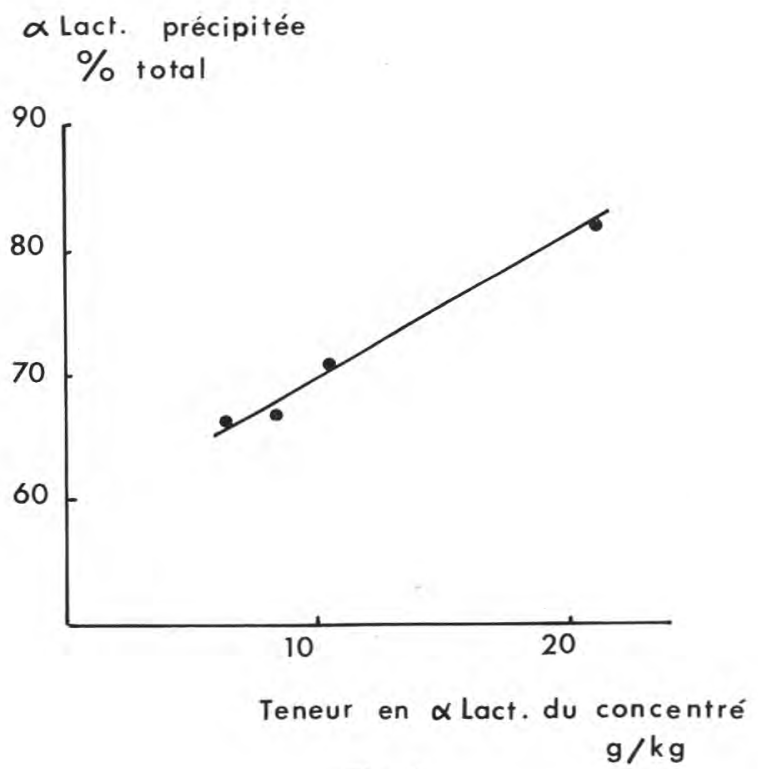

Fig. 5

Influence de la durée du traitement thermique sur la proportion d' $\alpha$-lactalbumine précipi-

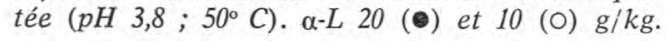

Time dependance of $\alpha$-lactalbumin precipitation $\left(p H 3.8 ; 50^{\circ} \mathrm{C}\right), \alpha-L 20(\bullet)$ and 10 (O) $\mathrm{g} / \mathrm{kg}$.

6. Optimisation des étapes du procédé de fractionnement

A partir des différents résultats obtenus, le protocole de séparation des protéines du lactosérum représenté figure 6 était défini. Les conditions retenues étaient celles correspondant au rendement de précipitation de $1^{\prime} \alpha$-lactalbumine le plus élevé sauf en ce qui concerne la température du traitement thermique $\left(55^{\circ} \mathrm{C}\right.$ au lieu de $65^{\circ} \mathrm{C}$ ) pour éviter toute dénaturation irréversible des protéines (PIERRE et al., 1977) et la nature de l'acide $\left(\mathrm{HCl}\right.$ au lieu de $\left.\mathrm{HNO}_{3}\right)$ pour limiter la pollution des eaux résiduaires par les ions nitrate.

\section{Préparation des fractions protéiques}

$500 \mathrm{~g}$ de rétentat de lactosérum contenant $132 \mathrm{~g} / \mathrm{kg}$ de $\beta$-lactoglobuline et $21 \mathrm{~g} / \mathrm{kg} \mathrm{d} \alpha$-lactalbumine étaient traités comme décrit figure 6. 


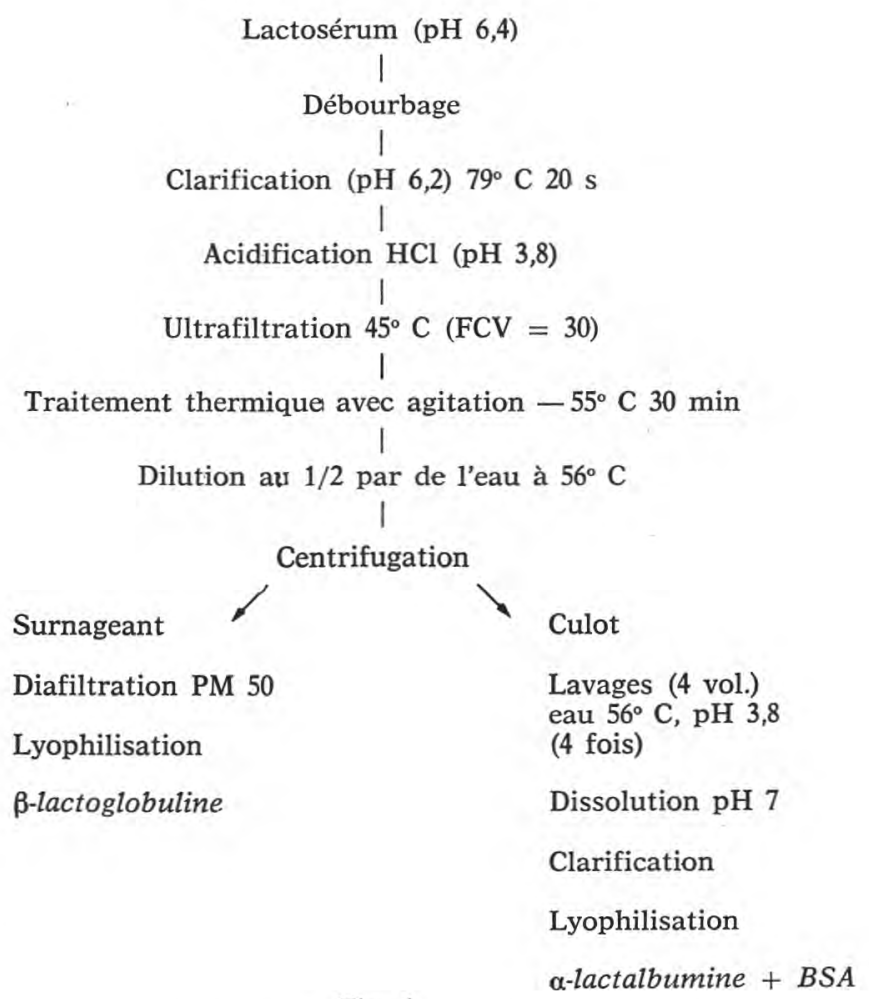

Fig. 6

Protocole de traitement du laciosérum pour la séparation des fractions protéiques Whey protein fractionation.

Le traitement thermique $\left(55^{\circ} \mathrm{C}, 30 \mathrm{~min}\right)$ conduisait à l'obtention d'un gel qui était dispersé dans $500 \mathrm{ml}$ d'eau distillée à $56^{\circ} \mathrm{C}$, le $\mathrm{pH}$ étant maintenu à 3,8 . La suspension était centrifugée à $1000 \mathrm{~g}$. La solution surnageante était prélevée et le culot conservé d'autre part.

\section{Isolement de la $\beta$-lactoglobuline}

Les teneurs en $\beta$-lactoglobuline et en $\alpha$-lactalbumine de la solution surnageante déterminées par HPLC étaient respectivement de 56 et $1,5 \mathrm{~g} / \mathrm{kg}$. Le pH était ajusté à 7,0 , puis la purification de la $\beta$-lactoglobuline était réalisée par ultrafiltration sur membrane PM 50 car la taille des pores de cette membrane est telle que 1' $\alpha$-lactalbumine et les solutés de faible poids moléculaire (lactose, sels minéraux, azote non protéique, etc.) ne sont pas retenus au contraire de la $\beta$-lactoglobuline. Une diafiltration avec de l'eau pure était réalisée jusqu'à ce que l'indice de réfraction du perméat ait été réduit de $90 \%$. Le rétentat final était lyophilisé et constituait la fraction $\beta$-lactoglobuline. La teneur en $\mathrm{Nx6,38}$ de la poudre était de $87 \%$. Le profil chromatographique (fig. 7) indique une pureté de la protéine voisine de $98 \%$, et l'électrophorèse (fig. 8) montre la présence d'un seul constituant protéique. 


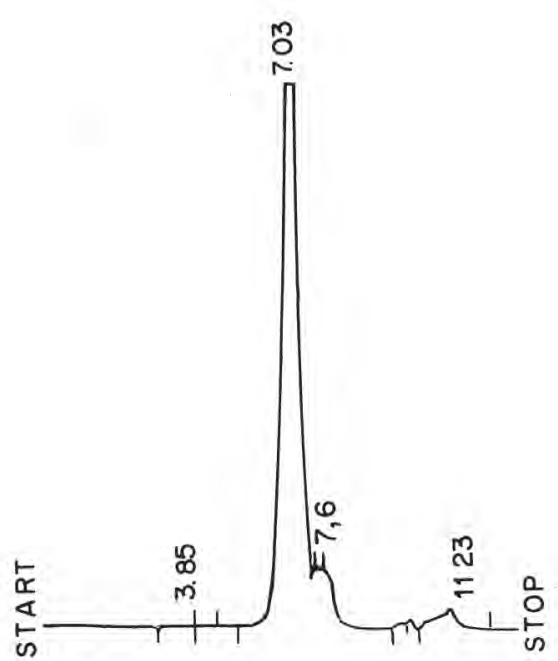

A

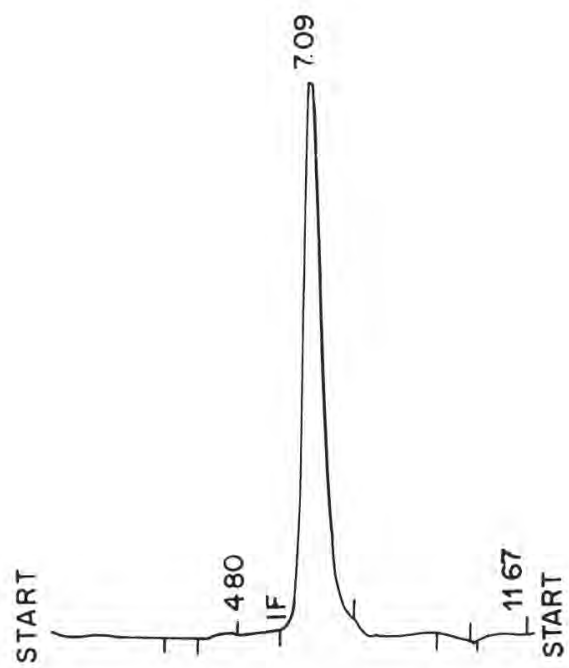

B

Fig. 7

Profil d'élution en HPLC de la fraction $\beta$-lactoglobuline avant $(A)$ et après $(B)$ purification. HPLC analysis of $\beta$-lactoglobulin fraction before $(A)$ and after $(B)$ purification.

\section{Purification de l' $\alpha$-lactalbumine}

Le culot de centrifugation était lavé à trois reprises par remise en suspension dans 4 volumes d'eau à $56^{\circ} \mathrm{C}$. Ces lavages avaient pour but d'éliminer les solutés de la phase aqueuse, en particulier la $\beta$-lactoglobuline. Le $\mathrm{pH}$ des eaux de lavage était ajusté à 3,8 , car une remontée du $\mathrm{pH}$ conduirait à une insolubilisation de la $\beta$-lactoglobuline en raison de la faible force ionique (AMUNDSON et al., 1982) qui, alors, ne serait plus éliminée par les lavages.

Le précipité ainsi lavé était repris dans un peu d'eau et le $\mathrm{pH}$ amené à 7,0 pour obtenir sa dissolution. Le profil d'élution en HPLC (fig. 9) montrait l'existence de plusieurs composants, le plus important étant l' $\alpha$-lactalbumine. La présence d'un pic correspondant aux lipoprotéines (V.E. : 4,6) amenait à tenter une nouvelle opération de clarification. La solution amenée à $\mathrm{pH} 7,0$ était additionnée de $1,2 \mathrm{~g} / 1$ de calcium sous forme de $\mathrm{CaCl}_{2}$ et portée à $55^{\circ} \mathrm{C}$ durant $10 \mathrm{~min}$. Le précipité observé était séparé par centrifugation et sur le surnageant, le pic correspondant aux lipoprotéines était notablement réduit (fig. 9).

La solution surnageante était lyophilisée. Cette «fraction $\alpha$-lactalbumine » contenait $78 \%$ de protéines $(\mathrm{Nx} 6,38)$ dont $50 \%$ étaient dosés comme de l' $\alpha$ lactalbumine par HPLC et $15 \%$ comme de la bovine sérum albumine. Les $13 \%$ non identifiés peuvent correspondre au pic élué dans l'exclusion en HPLC et représentant un matériel à haut poids moléculaire, ou polymérisé. L'électrophorèse confirme la présence de l' $\alpha$-lactalbumine et de la bovine sérum albumine 


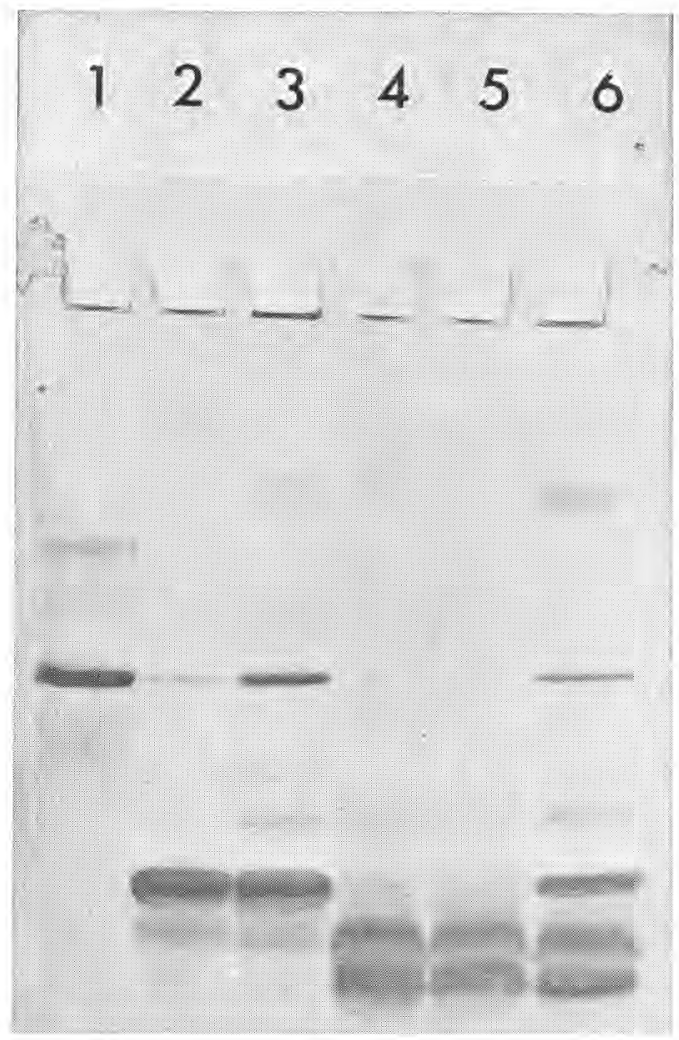

Fig. 8

Séparation par électrophorèse des constituants des fractions purifiées : fraction $\alpha$-lactalbumine (3) et $\beta$-lactoglobuline (4). Protéines de référence : 1 . Bovine sérum albumine; 2. $\alpha$-lactalbumine ; 5. $\beta$-lactoglobuline ; 6 . Lactosérum clarifié.

Electrophoresis of separated proteins : $\alpha$-lactalbumin fraction (3) and p.-lactoglobulin fraction (4). Reference proteins : 1. Bovine serum albumin ; 2 . $\alpha$-lactalbumin ; 5 . $\beta$-lactoglobulin; 6. Clarified whey.

comme constituants principaux (fig. 8). Aucune trace de $\beta$-lactoglobuline n'était décelée par les méthodes analytiques utilisées.

Dans le but de préparer une extrapolation industrielle du procédé, le lavage du précipité par microfiltration a été tenté sur $5 \mathrm{~kg}$ de rétentat de lactosérum ( $\beta$-lactoglobuline $100 \mathrm{~g} / \mathrm{kg}, \alpha$-lactalbumine $17 \mathrm{~g} / \mathrm{kg}$ ) traité thermiquement à $\mathrm{pH} 3,8$ et à $55^{\circ} \mathrm{C} 30 \mathrm{~min}$, puis dilué à $1 / 5$.

La figure 10 indique les dilutions résultant de la diafiltration par l'eau ainsi que l'évolution de la composition des rétentats et des microfiltrats au cours des lavages. Ce type de technologie semble efficace pour réduire la concentration de la $\beta$-lactoglobuline; par contre, il ne permet pas de l'éliminer totalement et, donc, la centrifugation sera nécessaire pour terminer le lavage du précipité. 


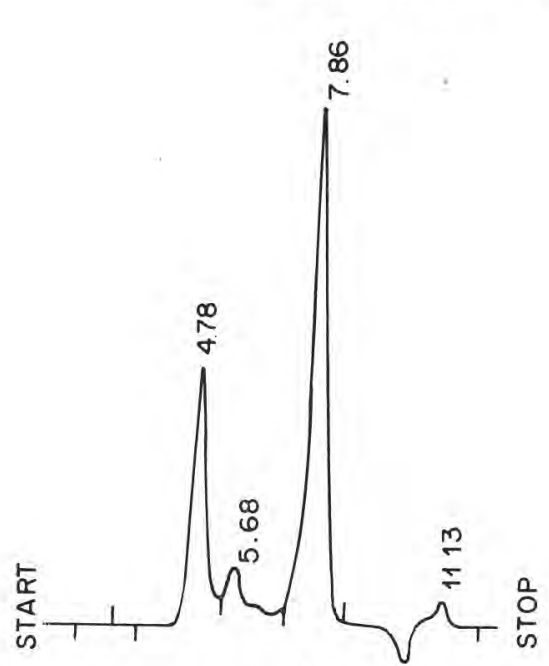

A

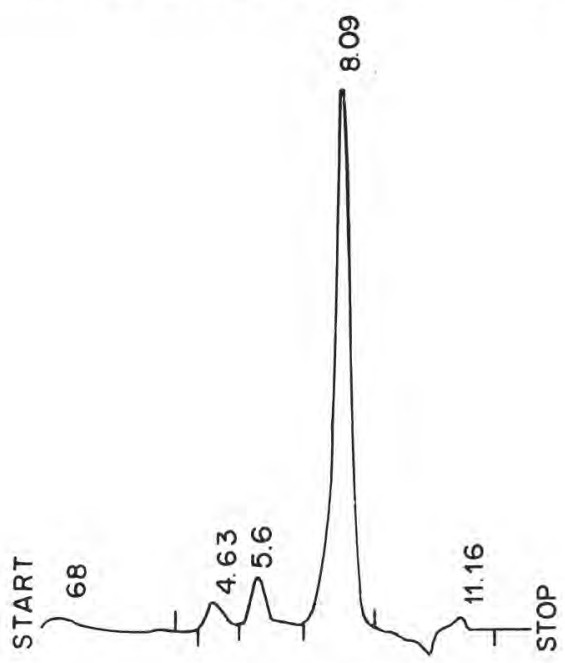

B

Fig. 9

Profil d'élution en HPLC de la fraction $\alpha$-lactalbumine avant $(A)$ et après $(B)$ clarification. VE 5,6 BSA; 7,9 $\alpha$-lactalbumine.

HPLC analysis of $\alpha$-lactalbumin fraction before $(A)$ and after $(B)$ clarifying treatment. EV 5.6 BSA ; $7.9 \alpha$-lactalbumin.

\section{Conclusion}

Le principe de séparation utilisé permet de réaliser avec des moyens technologiques simples le fractionnement des protéines majeures du lactosérum. La $\beta$-lactoglobuline est obtenue pure à $98 \%$ et le rendement de préparation est de l'ordre de $90 \%$ d'après les estimations que nous avons faites. L' $\alpha$-lactalbumine est obtenue en mélange avec la bovine sérum albumine. Etant donné la similitude des propriétés chimiques de ces protéines, il semble difficile de les séparer par des méthodes basées sur une activité physico-chimique. En raison de leur différence de taille moléculaire, il serait possible de faire appel soit au principe de gel filtration, soit à une ultrafiltration sur une membrane de porosité choisie. Une autre possibilité est d'allonger la durée du traitement thermique de la deuxième clarification jusqu'à $60 \mathrm{~min}$ environ de manière à dénaturer thermiquement la bovine sérum albumine et à la précipiter.

Le rendement en $\alpha$-lactalbumine est limité d'une part par l'équilibre de polymérisation de l' $\alpha$-lactalbumine, une partie de la protéine restant toujours à l'état monomère ( $5 \%$ environ dans les meilleures conditions); d'autre part, par le lavage du précipité, pendant lequel il faut respecter les conditions maintenant la polymérisation. Les pertes seraient de l'ordre de $20 \%$ à ce stade. Il est sans doute possible d'améliorer encore ce point de la technique. 

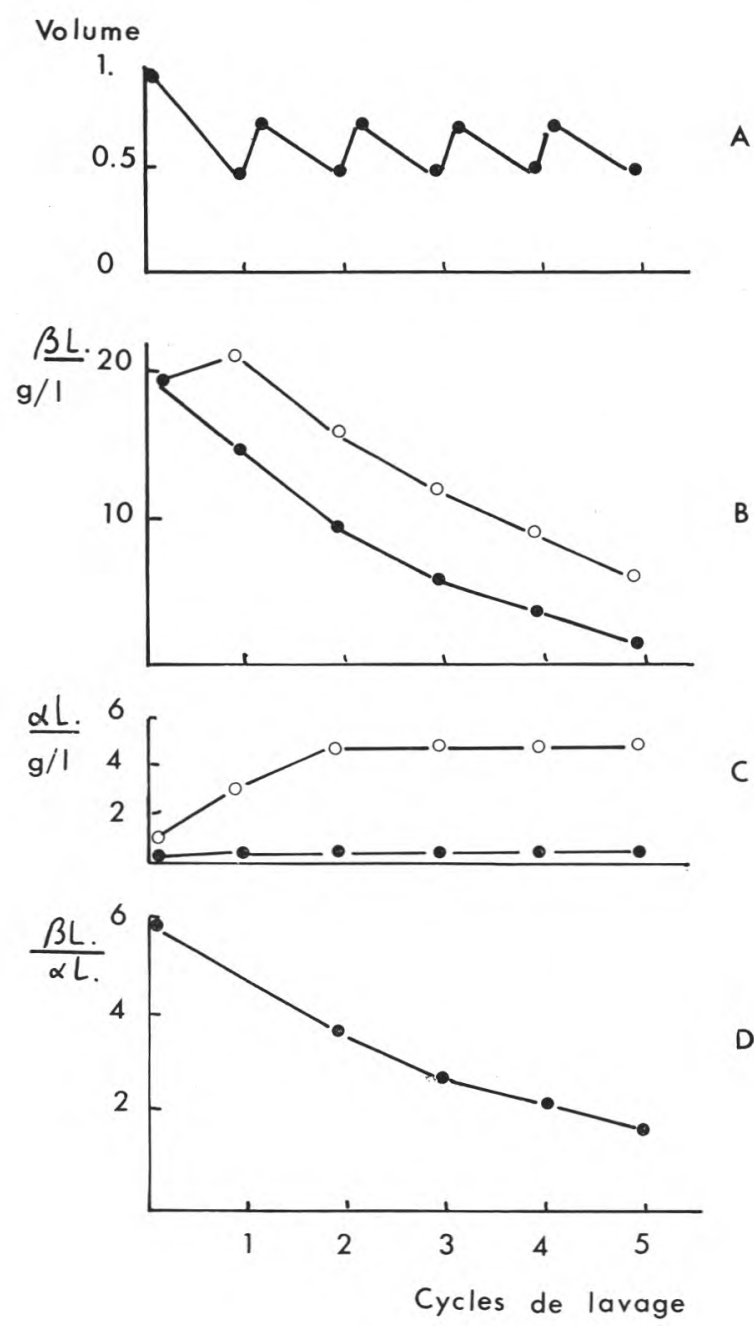

Fig. 10

Etude du lavage du précipité d' $\alpha$-lactalbumine par microfiltration. A : volume de diafiltration ; évolution de la teneur $(\mathrm{g} / \mathrm{kg})$ en $\beta$-lactoglobuline $(B)$ et en $\alpha$-lactalbumine $(C)$ $d u$ rétentat $(O)$ et du microfiltrat $(\bullet)$; $(D)$ évolution du rapport $\beta$-lactoglobuline/ $\alpha$-lactalbumine dans le rétentat.

Purification of $\alpha$-lactalbumin by microfiltration. A : Evolution of retentate volume during diafiltration cycles; $\beta$-lactoglobulin $(B)$ and $\alpha$-lactalbumin $(C)$ content $(\mathrm{g} / \mathrm{kg})$ of retentate $(0)$ and microfiltrate $(\bullet) ; D$ : $\beta$-lactoglobulin on $\alpha$-lactalbumin ratio in retentates.

La fraction contaminant l' $\alpha$-lactalbumine et la bovine sérum albumine n'a pas été identifiée. Par ailleurs, nous n'avons pas étudié la répartition des protéines mineures du lactosérum au cours du traitement. 


\section{Remerciements}

Nous remercions J.L. MAUBOIS pour l'intérêt qu'il a porté au déroulement de notre travail et pour la lecture critique du manuscrit.

Reç le 15 mai 1986.

Accepté pour publication le 26 juin 1986.

\section{Références bibliographiques}

Amundson C.H., Watanawani-Chakorn S., Hill Hr. C.G., 1982. Production of enriched protein fractions of $\beta$-lactoglobulin and $\alpha$-lactalbumin from cheese whey. $J$. Food Process. Preserv., 6, 55-71.

ANDREWS A.T., 1983. Proteinases in normal bovine milk and their action on caseins. J. Dairy Res., 50, 45-55.

Von Brantl V., Teschemacher H., 1982. Opiatartig wirkende stoffe in milch und milchproduktar. Milchwissenschaft, 37, 581-583.

EleanOR M., BRown, 1984. Interactions of $\beta$-lactoglobulin and $\alpha$-lactalbumin with lipids. A review. J. Dairy Sci., 67, 713-722.

Fauquant J., Pierre A., Brulé G., 1985. Clarification du lactosérum acide de caséinerie. Tech. Lait. (1003), 37-41.

Fauquant J., Vieco E., Brulé G., Maubors J.L., 1985. Clarification des lactosérums doux par agrégation thermocalcique de la matière grasse résiduelle. Lait, 65 (1), 1-20.

HaRris P., Yoell R.W., 1985. Process preparation of soluble preparation and use. United States Patent, US 45282203.

KRONMAN M.J., ANDREOTti R., Vitols R., 1964. Inter and intra-molecular interactions of $\alpha$-lactalbumin. II. Aggregation reactions at acid pH. Biochemistry, 3 (8), 1152-1160.

Kumata T., Pham A.M., Ma C.Y., NaKax S., 1985. Elimination of $\beta$-lactoglobulin from whey to simulate human milk protein. J. Food Sci., 50, 605-609.

Maubors J.L., 1982. Les protéines du lactosérum extraites par ultrafiltration. In : Protéines animales, extraits concentrés et isolats en alimentation humaine. Bourgeois C.M. et Le Roux P., Ed. Apria-Lavoisier, 172-190.

MaUbors J.L., 1984. Separation, extraction and fractionation of milk protein components. Lait, 64, 485-495.

Mrrabel B., 1978. Nouveau procédé d'extraction des protéines du lactosérum. Ann. Nutr. Aliment., 32, 243-253.

Nielsen M.A., Coulter S.T., MoRR C.V., Rosenau J.R., 1973. - Four factor response surface experimental design for evaluating the role of processing variables upon proteins denaturation in heated whey systems. J. Dairy Sci., 56, 76-83.

PEARCE R.J., 1983. Thermal separation of $\beta$-lactoglobulin and $\alpha$-lactalbumin in bovine Cheddar cheese whey. Aust. J. Dairy Technol., 38, 144-149.

Pierre A., Brulé G., Fauquant J., Piot M., 1977. Influence des traitements thermiques sur les propriétés physicochimiques des rétentats obtenus par ultrafiltration de lait de vache et de lait de chèvre. I. Dénaturation des produits solubles. Lait, 57, 646-662.

ROGER L., 1979. Contribution à la recherche d'une meilleure utilisation en alimentation humaine des composants glucidiques et protéiques du lactosérum grâce à l'emploi des techniques à membrane. Thèse Université de Rennes.

SKUDDER P.J., 1985. Evaluation of a porous silica-based ion exchange medium for the production of protein fractions from rennet and acid-whey. J. Dairy Res., 52, 167-181. 